\title{
Risque d'éclosions de COVID-19 et de décès de résidents dans les foyers de soins de longue durée à but lucratif
}

\author{
Nathan M. Stall MD, Aaron Jones MSc PhD, Kevin A. Brown MSc PhD, Paula A. Rochon MD MSP, Andrew P. Costa PhD
}

Citation : CMAJ 2020 August 17;192:E946-55. doi : 10.1503/cmaj.201197-f; diffusion hâtive le 27 mai 2020

Entrevue avec l'auteur (en anglais) en baladodiffusion : https://soundcloud.com/cmajpodcasts/201197-res

Voir la version anglaise de l'article ici : www.cmaj.ca/lookup/doi/10.1503/cmaj.201197; voir le commentaire connexe en anglais ici : www.cmaj. ca/lookup/doi/10.1503/cmaj.201714; voir le commentaire connexe en français ici : www.cmaj.ca/lookup/doi/10.1503/cmaj.201714-f

\section{RÉSUMÉ}

CONTEXTE : Les foyers de soins de longue durée (SLD) ont jusqu'à présent été l'épicentre de la pandémie de maladie à coronavirus 2019 (COVID-19) au Canada. Selon des études antérieures, les soins offerts dans les foyers de SLD à but lucratif sont de qualité inférieure pour toute une gamme d'indicateurs de résultats et de processus, ce qui soulève la question suivante : les conséquences de la COVID-19 ont-elles été pires dans les foyers à but lucratif que dans ceux à but non lucratif?

MÉTHODES : Une étude de cohorte rétrospective basée sur l'ensemble des foyers de SLD en Ontario a été menée pour la période du 29 mars au 20 mai 2020 à partir de la base de données sur les éclosions de COVID-19 alimentée par le ministère des Soins de longue durée de l'Ontario. Des méthodes logistiques hiérarchiques et basées sur des données de comptage ont été utilisées pour modéliser les associations entre le statut financier des foyers de SLD (à but lucratif, à but non lucratif ou municipal) et les éclosions de
COVID-19 dans ces derniers, l'ampleur des éclosions (nombre de résidents infectés) et le nombre de décès de résidents attribuables à la COVID-19.

RÉSULTATS : L'analyse portait sur les 623 foyers de SLD de l'Ontario, qui comptent 75676 résidents. Parmi ces foyers, $360(57,7 \%)$ sont à but lucratif; $162(26,0 \%)$ sont à but non lucratif; et $101(16,2 \%)$ sont des foyers municipaux. Au total, 190 (30,5\%) éclosions de COVID-19 ont été enregistrées dans des foyers de SLD. Elles ont touché 5218 résidents et entraîné 1452 décès, ce qui représente un taux de létalité général de $27,8 \%$. Les probabilités d'une éclosion dans un foyer ont été associées à l'incidence de la COVID-19 dans la circonscription sanitaire entourant celui-ci (rapport de cotes [RC] ajusté 1,91 ; intervalle de confiance [IC] à $95 \%$ 1,19-3,05), au nombre de résidents dans l'établissement (RC ajusté 1,38; IC à $95 \% 1,18-1,61$ ) et à l'application des anciennes normes d'aménagement (RC ajusté 1,55 ; IC à $95 \% 1,01-2,38$ ), mais pas au statut financier d'un foyer. Comparativement au statut "à but non lucratif », le statut « à but lucratif » a été associé à l'ampleur d'une éclosion dans un foyer de SLD (risque relatif [RR] 1,96; IC à $95 \%$ 1,26-3,05) ainsi qu'au nombre de décès de résidents (RR ajusté 1,78 ; IC à $95 \%$ 1,03-3,07). Ces associations s'expliquent par une plus grande prévalence des anciennes normes d'aménagement dans les foyers de SLD à but lucratif ainsi qu'à l'appartenance à une chaîne de propriétés.

INTERPRÉTATION : Le statut «à but lucratif » est associé à l'ampleur d'une éclosion de COVID-19 et au nombre de décès de résidents dans un foyer de SLD, mais pas au risque d'éclosion. Deux principaux facteurs expliquent les différences entre les foyers à but lucratif et non lucratif, soit l'application des anciennes normes d'aménagement et l'appartenance à une chaîne de propriétés. Ceux-ci devraient être au cœur des futures mesures et politiques de lutte contre les infections.

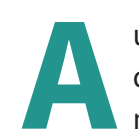

u Canada, les foyers de soins de longue durée (SLD) sont devenus l'épicentre de la pandémie de maladie à coronavirus 2019 (COVID-19). En effet, les résidents de ces établissements comptent pour plus de $80 \%$ des décès enregistrés au pays ${ }^{1-3}$. Les résidents des foyers de SLD sont plus à risque de contracter le coronavirus du syndrome respiratoire aigu sévère 2 (SRAS-CoV-2) en raison de leur mode de vie en collectivité et de leur exposition potentielle à des employés infectés au SRAS-CoV-2 pouvant être asymptomatiques ou présymptomatiques $^{4-6}$. Comme la plupart des personnes âgées, ces résidents affichent également un risque élevé de morbidité et de mortalité pour des raisons de fragilité et de multimorbidité ${ }^{7}$ L'inquiétude 
quant à la situation dans les foyers de SLD est généralisée étant donné qu'ils ont manqué de préparation et d'équipement pour bien protéger leurs résidents, et ce, malgré les prédispositions de ces derniers au risque. Des questions ont d'ailleurs été soulevées quant à l'ampleur des conséquences de la COVID-19 dans les foyers à but lucratif8.

En Ontario, la province canadienne la plus peuplée, tous les résidents des foyers de SLD se voient offrir des soins personnels et infirmiers ainsi qu'un logement subventionné dans le cadre d'un programme de SLD financé par les fonds publics. Nonobstant ce financement gouvernemental, chaque foyer de SLD peut être détenu et exploité par une entité à but lucratif, à but non lucratif ou municipale (publique ${ }^{10}$. Selon plusieurs études observationnelles, les soins offerts dans les foyers à but lucratif sont généralement de qualité inférieure pour toute une gamme d'indicateurs de résultats et de processus ${ }^{11,12}$ : notons entre autres un personnel insuffisant ou moins bien formé, davantage de plaintes des résidents et des familles, des taux plus élevés de visites à l'urgence, un plus grand nombre d'hospitalisations en soins actifs ainsi que des taux de mortalité plus élevés ${ }^{13-22}$. Selon des données américaines, les foyers à but lucratif seraient également plus susceptibles de recevoir des avis de non-respect des règles en matière de contrôle des infections et de pratiques d'hygiène des mains ${ }^{23,24}$. À la lumière de ces données et des conséquences catastrophiques de l'épidémie de COVID-19 dans les foyers de SLD, la présente étude s'est penchée sur les risques d'éclosions et de décès au plus fort de la pandémie dans les foyers ontariens en fonction de leur statut financier.

\section{Méthodes}

\section{Modèle d'étude}

Une étude de cohorte rétrospective portant sur l'ensemble des 623 foyers de SLD en Ontario a été menée pour la période du 29 mars 2020 - date à laquelle la première éclosion a été signalée dans un foyer en Ontario - au 20 mai 2020; cette période couvre la phase aiguë de l'épidémie de COVID-19 dans les établissements de SLD de la province ${ }^{25}$. Les maisons de retraite de l'Ontario n'ont pas été analysées dans la présente étude, car elles sont entièrement financées par des fonds privés et ne sont pas gérées par le ministère des Soins de longue durée. Les lignes directrices des déclarations STROBE (Strengthening the Reporting of Observational Studies in Epidemiology) et RECORD (Reporting of Studies Conducted Using Observational Routinely Collected Health Data) ont été suivies ${ }^{26,27}$.

\section{Sources de données et variables explicatives}

Toutes les données ont été obtenues auprès des ministères de la Santé et des Soins de longue durée de l'Ontario par l'intermédiaire du comité provincial de concertation sur la modélisation de la COVID-19 (Provincial COVID-19 Modelling Consensus Table). Parrainé par le ministère de la Santé de l'Ontario, Santé Ontario et Santé publique Ontario, ce comité est un groupe spécial bénévole formé de décideurs et de scientifiques chevronnés dont le mandat consiste à élaborer des estimations consensuelles et crédibles des répercussions de la
COVID-19. Certaines données relatives aux foyers, comme le nombre cumulatif de cas et de décès parmi les résidents, ont été fournies par la Direction de l'inspection des foyers de soins de longue durée. Ces données sont recueillies quotidiennement par des inspecteurs à l'échelle de la province, qui communiquent avec les foyers de SLD et colligent des données sur les éclosions dans un outil de suivi des cas de COVID-19. Cet outil contient les données des résidents décédés dans un foyer de SLD et de ceux qui occupaient toujours un lit dans un foyer, mais qui sont décédés ailleurs. Il n'inclut pas les données des résidents qui avaient obtenu leur congé d'un foyer de façon permanente et qui sont décédés ailleurs. La corrélation s'est révélée excellente entre les données de l'outil de suivi sur les cas et les décès et celles provenant d'autres sources provinciales. Pour le décompte des cas de COVID-19 dans les foyers de SLD, l'outil de suivi a été associé à des coefficients de corrélation de Pearson de 0,952 et de 0,914 avec les bases de données du Système intégré d'information sur la santé publique (SIISP) et du Système d'information de laboratoire de l'Ontario, respectivement ${ }^{28,29}$. En ce qui concerne les décès, l'outil a été associé à des coefficients de corrélation de Pearson de 0,913 et de 0,904 avec les bases de données du SIISP et du coroner, respectivement ${ }^{28}$. L'outil contient également de l'information sur le statut financier des foyers, c'est-à-dire s'ils sont à but lucratif (foyers privés exploités par le propriétaire ou rattachés à une chaîne constituée en société), à but non lucratif (organismes caritatifs, religieux ou communautaires) ou municipaux (foyers gérés par la municipalité; les employés sont des fonctionnaires municipaux) ${ }^{30}$.

D’autres données ont été fournies par le ministère des Soins de longue durée de l'Ontario, dont le nombre de lits autorisés; le nombre de résidents actifs en date du 31 mars 2020; la composition spécifique des types d'occupation (1, 2 ou 4 résidents par chambre); le ratio équivalent temps plein : lits; l'appartenance à une chaîne et la taille de celle-ci; et les normes d'aménagement en vigueur dans le foyer, qui se basent sur une classification structurale ${ }^{31}$. En 1972, la Loi sur les foyers de soins de longue durée de l'Ontario a été modifiée pour inclure des normes ciblant entre autres les bâtiments (elles permettaient 4 lits par chambre pourvue d'un lavabo et d'une toilette avec chasse d'eau; depuis la mise à jour des normes en 1998, seules les chambres individuelles et à 2 lits sont permises) ${ }^{30}$. Les foyers de SLD excédant les normes de 1972 ont été classés dans la catégorie "normes d'aménagement récentes ", tandis que ceux ne dépassant pas les normes de 1972 ont été classés dans la catégorie " anciennes normes d'aménagement ». Ces derniers ont des chambres plus petites, moins de chambres individuelles et davantage de salles de bain partagées (annexe 1, tableau S1, accessible en anglais au www.cmaj.ca/ lookup/doi/10.1503/cmaj.201197/tab-related-content).

L'incidence cumulative de la COVID-19 dans les circonscriptions sanitaires entourant chaque foyer a également été mesurée à partir des données individuelles dépersonnalisées de tous les cas confirmés de COVID-19 (en date du 19 mai 2020) provenant de la base de données du SIISP. L'objectif consistait à calculer le taux de cas de COVID-19 par millier d'habitants (à partir des données provenant du recensement de 2016) pour chacune des 
35 circonscriptions sanitaires de l'Ontario desservies par un bureau de santé publique ${ }^{28}$. Les résidents des foyers de SLD ont été exclus du numérateur et du dénominateur dans les calculs d'incidence. Le nombre d'habitants dans les collectivités abritant les foyers a été calculé à l'aide du Fichier de conversion des codes postaux plus de Statistique Canada et à partir des codes postaux de la Société canadienne des postes utilisés jusqu'en novembre 2018 inclusivement. Les collectivités de moins de 10000 habitants ont été classées dans la catégorie " rurales ».

\section{Exposition et paramètres principaux}

L'exposition d'intérêt principale était le statut financier des foyers de SLD (à but lucratif, à but non lucratif ou municipal), ce qui explique pourquoi l'analyse a été menée à l'échelle des foyers et non des résidents. Les principaux paramètres d'intérêt étaient les éclosions de COVID-19 dans les foyers (au moins 1 cas parmi les résidents), l'ampleur des éclosions (nombre total de cas confirmés parmi les résidents des foyers en éclosion) et le nombre total de décès de résidents attribuables à la COVID-19 (dans les foyers ayant une éclosion).

\section{Analyse statistique}

Des statistiques sommaires ont servi à comparer les caractéristiques des foyers de SLD ainsi que leurs nombres de cas de COVID-19 et de décès de résidents en fonction de leur statut financier. Un test $\chi^{2}$ a été utilisé pour les variables nominales, et un test de Kruskal-Wallis, pour les variables continues. Un test binomial exact a servi à comparer l'incidence de la COVID-19 dans les 35 circonscriptions sanitaires entourant les foyers de la province. Le taux de létalité a été calculé en mesurant la proportion de résidents décédés de la COVID-19 parmi tous les résidents infectés.

Un modèle statistique multivariable à barrière (hurdle) a été élaboré pour chacun des 3 paramètres d'intérêt. Le risque d'éclosion de COVID-19 dans un foyer comptant au moins un résident infecté a été modélisé au moyen d'une régression logistique étant donné la nature dichotomique des résultats. L'ampleur des éclosions de COVID-19 et le nombre total de décès de résidents ont été modélisés au moyen d'une régression quasi-Poisson avec une variable de décalage pour tenir compte du nombre de résidents actifs dans un foyer donné. En ce qui a trait à l'ampleur des éclosions, le modèle a pris en considération la surdispersion des résultats par rapport aux valeurs de comptage habituelles de la loi de Poisson. Des points d'interception aléatoires correspondant à chaque circonscription sanitaire ont été inclus dans les modèles ${ }^{33}$. Trois modèles ont été créés pour chacun des 3 paramètres d'intérêt, et les mêmes facteurs de correction ont été appliqués.

Le modèle $n^{\circ} 1$ inclut seulement le statut financier des foyers (non corrigé). Le modèle $\mathrm{n}^{\circ} 2$ inclut le statut financier des foyers et les caractéristiques de leur circonscription sanitaire (nombre d'habitants dans la collectivité; nombre de cas de COVID-19 par 1000 habitants dans la circonscription sanitaire). Ces covariables ont été considérées comme des facteurs extrinsèques aux foyers de SLD. Le modèle $n^{\circ} 3$ est explicatif et inclut le statut financier et les caractéristiques de la circonscription sanitaire des foyers ainsi que des facteurs propres à ces derniers (nombre de résidents actifs, normes d'aménagement en vigueur, ratio employé : lits, appartenance à une chaîne de foyers et taille de celle-ci). Ces covariables ont été considérées comme des facteurs intrinsèques aux foyers de SLD.

Le modèle $n^{\circ} 2$ a été utilisé dans un premier temps pour tirer les principales conclusions, tandis que le modèle $n^{0} 3$ a servi à cibler les facteurs explicatifs ayant un lien avec le statut financier des foyers. Le type d'occupation des chambres n'a pas été retenu dans les modèles finaux en raison d'une association colinéaire avec les normes d'aménagement en vigueur dans les foyers (coefficient de corrélation de Pearson : 0,81). Les normes d'aménagement en vigueur ont plutôt été choisies comme covariable d'intérêt puisqu'elles reflètent non seulement l'occupation des chambres (seules les anciennes normes permettaient des chambres à 4), mais aussi d'autres facteurs comme la taille des espaces communs, des chambres et des salles de bains partagées (annexe 1, tableau S1). À l'étape de l'analyse de sensibilité, les 3 modèles pour chaque paramètre ont été réanalysés en remplaçant les normes d'aménagement en vigueur par l'occupation moyenne des chambres (annexe 1, tableau S2). Des graphiques en essaim d'abeilles ont servi à représenter la distribution de l'ampleur des éclosions de COVID-19 et du nombre total de décès dans les foyers en fonction du statut financier, des normes d'aménagement en vigueur et de l'appartenance des foyers à une chaîne.

Toutes les covariables ont été initialement sélectionnées à l'aide d'une revue de littérature et de l'opinion d'experts d'épidémiologistes spécialisés en maladies infectieuses et de spécialistes en gériatrie et en SLD. Les analyses ont été effectuées à l'aide de la version 9.4 du logiciel statistique SAS (SAS Institute Inc.). Les tests effectués étaient bilatéraux, et un seuil de signification statistique de $\alpha=0,05$ a été appliqué.

\section{Approbation éthique}

Cette étude a été approuvée par le comité d'éthique de la recherche de l'Université de Toronto et par le comité d'éthique de la recherche intégré de Hamilton.

\section{Résultats}

L'analyse portait sur les 623 foyers de SLD de l'Ontario, qui comptent 75676 résidents. Parmi ces foyers, 360 (57,7\%) sont à but lucratif; $162(26,0 \%)$ sont à but non lucratif; et 101 (16,2\%) sont des foyers municipaux (tableau 1). En comparaison avec les foyers municipaux et à but non lucratif, les foyers à but lucratif étaient généralement plus petits (nombre moyen de lits autorisés plus faible), appliquaient davantage les anciennes normes d'aménagement et affichaient la plus faible proportion de chambres individuelles ainsi que la plus grande proportion d'appartenance à une chaîne.

Au total, des éclosions de COVID-19 ont été recensées dans $190(30,4 \%)$ foyers de SLD en Ontario avec une incidence cumulative brute de 66,4 par 1000 personnes. L'incidence cumulative des éclosions de COVID-19 était de 85,1 par 1000 résidents dans les foyers à but lucratif; de 61,4 par 1000 résidents dans les foyers à but non lucratif; et de 23,4 par 1000 résidents dans les foyers municipaux (tableau 2). Le taux de mortalité brut lié à la COVID19 se fixait à 23,4 par 1000 résidents dans les foyers à but lucratif; 
Statut financier des foyers de SLD

\section{Indicateur}

Nombre moyen de résidents

Anciennes normes d'aménagement ${ }^{\star \star}$

Type de chambre

$\%$ de chambres individuelles, moyenne

$\%$ de chambres à 2 , moyenne

$\%$ de chambres à 4 , moyenne

Chaîne de foyers de SLD

$\geq 20$ foyers

Entre 10 et 19 foyers

Entre 2 et 9 foyers

1 foyer (pas une chaîne)

Ratio employés (équivalents temps plein) :

lits, moyenne

Nombre d'habitants dans la collectivité abritant le foyer de SLD

$$
\begin{aligned}
& \geq 500000 \\
& 10000-499999
\end{aligned}
$$

$<10000$ (rurale)

Incidence cumulative de la COVID-19 dans les circonscriptions sanitaires entourant les foyers de SLD

\section{Nombre $(\%)^{\star}$ de foyers à but non lucratifł $n=162$}

$30(18,5)$

$(11,9)$

$<0,001$

$193(53,6)$

49,2

39,9

52,8

$<0,001$

38,5

8,7

39,7

0,52

28,3

$0(0)$

$0(0)$

$104(28,9)$

$50(30,9)$

$112(69,1)$

0,72

6,6

$<0,001$

$56(15,6)$

$55(15,3)$

0,70

$0(0)$

$<0,001$

$0(0)$

$0(0)$

$101(100)$

0,77

$<0,001$
$82(50,6)$
$37(22,8)$
$43(26,5)$
1,06 par 1000

1,00 par 1000

$28(27,7)$
$45(44,5)$
$28(27,7)$
$0,89 \operatorname{par} 1000$

0,002

0,045

Remarque : COVID-19 = maladie à coronavirus 2019; SLD = soins de longue durée

*Sauf indication contraire.

†Nombre de résidents actifs dans les foyers de SLD à but lucratif en Ontario en date du 31 mars $2020: 40752 ;$ nombre de lits : 42310.

$\ddagger$ Nombre de résidents actifs dans les foyers de SLD à but non lucratif en Ontario en date du 31 mars $2020: 19375 ;$ nombre de lits : 20181.

§Nombre de résidents actifs dans les foyers de SLD municipaux en Ontario en date du 31 mars $2020: 15$ 675; nombre de lits : 12269.

ITest $\chi^{2}$ pour les variables nominales et test de Kruskal-Wallis pour les variables continues, $\alpha=0,05$.

*Foyers de SLD dans lesquels $\geq 50 \%$ des lits ne dépassent pas les normes d’aménagement de 1972 (annexe 1, tableau S1).

à 18,2 par 1000 résidents dans les foyers à but non lucratif; et à 5,8 par 1000 résidents dans les foyers municipaux. Le taux de létalité chez les résidents se chiffrait à $27,5 \%$ dans les foyers à but lucratif; à 29,7\% dans les foyers à but non lucratif; et à $25,0 \%$ dans les foyers municipaux.

\section{Probabilités d'une éclosion de COVID-19 dans les foyers de SLD}

En tout, 190 (30,5 \%) foyers de SLD de l'Ontario ont vécu une éclosion de COVID-19, dont 110 (30,6\%) foyers à but lucratif; 55 $(34,0 \%)$ foyers à but non lucratif; et $25(24,8 \%)$ foyers municipaux (tableau 2). Dans les modèles de régression logistique $n^{\circ} 1$ (sans correction) et $\mathrm{n}^{\circ} 2$ (corrigé pour tenir compte des caractéristiques de la circonscription sanitaire), aucune corrélation significative n'a été établie entre le statut financier d'un foyer et les probabilités d'une éclosion de COVID-19 (tableau 3). Dans le modèle explicatif $\mathrm{n}^{\circ} 3$ (entièrement corrigé), ce paramètre a été associé de façon significative à l'incidence cumulative de la COVID-19 dans la circonscription sanitaire entourant le foyer (rapport de cotes [RC] ajusté 1,91; intervalle de confiance à $95 \%$ [IC] 1,19-3,05; par augmentation de 1/1000 de l'incidence cumulative de la COVID-19); au nombre total de résidents actifs (RC ajusté 1,38; IC à $95 \% 1,18-$ 1,61 ; par 50 lits); et à l'application des anciennes normes d'aménagement (RC ajusté 1,55; IC à $95 \%$ 1,01-2,38).

\section{Ampleur des éclosions de COVID-19 dans les foyers de SLD}

Parmi les foyers de SLD ayant connu une éclosion de COVID-19, la proportion moyenne de résidents infectés se chiffrait à 23,8\% dans les foyers à but lucratif comparativement à $17,2 \%$ et à $7,1 \%$ dans les foyers à but non lucratif et municipaux respectivement (tableau 2). Parmi les 15 foyers associés aux taux d'incidence les plus élevés, 12 étaient des foyers à but lucratif appliquant les anciennes normes d'aménagement et appartenant à une chaîne (figure 1). Dans les modèles de régression quasi-Poisson sans correction (risque relatif [RR] 1,83; IC à $95 \% 1,18-2,84$ ) et corrigé pour tenir compte des caractéristiques des circonscriptions sanitaires (RR ajusté 1,96; IC à $95 \% 1,26-3,05)$, le statut « à but 
Tableau 2 : Éclosions de COVID-19 et décès dans les foyers de SLD en Ontario en fonction du statut financier (du 29 mars au 20 mai 2020)

Statut financier des foyers de SLD

Nombre (\%)* de

foyers à but lucratif $\dagger$ $n=360$

Nombre $(\%)^{\star}$ de foyers
à but non lucratif $\neq$
$n=162$

Nombre (\%)* de

foyers municipaux $\$$ $n=101$
Valeur de $p$

Éclosions de COVID-19

Toutes éclosions confondues dans un foyer de SLD

Éclosions ayant touché des résidents et

des employés

Éclosions ayant touché des résidents seulement

Éclosions ayant touché des employés seulement

Éclosions de COVID-19 ayant touché des résidents

Nombre total de cas de COVID-19

Incidence cumulative des cas de COVID-19

Nombre de foyers avec une éclosion chez

les résidents

Pourcentage de résidents infectés par foyer en éclosion, médiane (EI)

Nombre de cas par foyer en éclosion, médiane (EI)

Décès de résidents liés à la COVID-19

Nombre total de décès liés à la COVID-19

Taux de mortalité de la COVID-19

Foyers comptant au moins un décès de résident

Pourcentage de décès de résidents par foyer, médiane (EI)

Nombre médian de décès par foyer (EI)

Taux de létalité (\%)

\section{$154(42,8)$}

$51(14,2)$

$73(45,1)$

$29(17,9)$

$59(16,4)$

$44(12,2)$

3599

85,1 par 1000

$110(30,6)$

$4,8(1,1-49,6)$

5 (1-55)

989

23,4 par 1000

$51(14)$

$13,0(5,1-19)$

14 (8-27)

27,5
$26(16,0)$

$18(11,1)$

1239

61,4 par 1000

$55(34,0)$

$5,6(1,5-33,8)$

$10(1-35)$

368

18,2 par 1000

33 (20)

$7,0(2,4-12)$

$10(4-16)$

29,7
$41(40,6)$

$13(12,9)$

0,77

0,44

$12(11,9)$

0,53

$16(15,8)$

0,51

380

23,4 par 1000

$25(24,8)$

$<0,001$

0,29

$1,1(0,6-4,6)$

0,01

$2(1-10)$

0,20

95

5,8 par 1000

$<0,001$

11 (11)

0,086

$2,3(1,0-6,8)$

0,0019

$3(2-13)$

0,013

25,0

0,14

Remarque : COVID-19 = maladie à coronavirus 2019; EI = écart interquartile; SLD = soins de longue durée.

*Sauf indication contraire.

†Nombre de résidents actifs dans les foyers de SLD à but lucratif en Ontario en date du 31 mars 2020:40 752; nombre de lits : 42310.

¥Nombre de résidents actifs dans les foyers de SLD à but non lucratif en Ontario en date du 31 mars $2020: 19375 ;$ nombre de lits : 20181.

$\S$ Nombre de résidents actifs dans les foyers de SLD municipaux en Ontario en date du 31 mars 2020:15 675; nombre de lits : 12269.

१Test binomial exact pour l'incidence, test $\chi^{2}$ pour les variables nominales et test de Kruskal-Wallis pour les variables continues, $\alpha=0,05$.

lucratif » a été associé de façon significative à l'ampleur d'une éclosion dans un foyer (nombre total de résidents infectés) comparativement au statut «à but non lucratif » (tableau 4). Le risque associé au statut financier « à but lucratif » était encore plus grand lorsque les foyers municipaux étaient utilisés comme catégorie de référence dans le modèle (annexe 1 , tableau S3).

Dans le modèle explicatif entièrement corrigé, l'association entre l'ampleur d'une éclosion dans un foyer et le statut «à but lucratif » était moins forte (RR ajusté 0,96; IC à $95 \% 0,57-1,61$ ). Ce paramètre a toutefois été associée de façon significative avec le nombre de cas de COVID-19 par 1000 habitants dans la circonscription sanitaire entourant un foyer (RR ajusté 1,65; IC à $95 \%$ 1,02-2,67), l'application des anciennes normes d'aménagement (RR ajusté 1,88; IC à $95 \%$ 1,27-2,79) et l'appartenance à une chaîne (RR ajusté 1,84; IC à 95 \% 1,08-3,15). Le nombre total de résidents actifs dans un foyer constituait pour sa part un facteur de protection (RR ajusté 0,84; IC à $95 \%$ 0,73-0,95; par 50 lits).
Nombre de décès de résidents liés à la COVID-19 dans les foyers de SLD en éclosion

Dans les foyers ayant connu une éclosion, la proportion moyenne de résidents décédés de la COVID-19 se chiffrait à 6,5\% dans les foyers à but lucratif comparativement à 5,5\% et à 1,7\% dans les foyers à but non lucratif et municipaux, respectivement (tableau 2). Parmi les 10 foyers comptant les plus grands nombres de décès, 7 étaient des foyers avec but lucratif appliquant les anciennes normes d'aménagement et appartenant à une chaîne (figure 2). Le statut « à but lucratif » a été associé au nombre total de décès de résidents liés à la COVID-19 dans le modèle de régression quasiPoisson corrigé pour tenir compte des caractéristiques de la circonscription sanitaire (RR ajusté 1,78; IC à $95 \%$ 1,03-3,07), mais pas dans celui sans correction (RR 1,67; IC à $95 \%$ 0,99-2,73) (tableau 5). Une fois de plus, le risque associé au statut financier « à but lucratif » était encore plus grand lorsque les foyers municipaux étaient utilisés comme groupe de référence (annexe 1, tableau S3). 
Tableau 3 : Probabilités d'éclosion de COVID-19 dans les foyers de SLD en fonction du statut financier

Modèle 1
(statut financier
seulement),
RC ajusté
(IC à $95 \%$ )

Variable

\section{Statut financier}

À but non lucratif (Réf.)

À but lucratif

Municipal

\section{Caractéristiques de la circonscription sanitaire}

Incidence cumulative de la COVID-19 dans la circonscription sanitaire (1 cas par 1000 habitants)

Nombre d'habitants

$$
\begin{aligned}
& \geq 500000 \text { (Réf.) } \\
& 10000-499999 \\
& <10000 \text { (rural) }
\end{aligned}
$$

\section{Caractéristiques du foyer de SLD}

Nombre de résidents (par unité de 50)

Anciennes normes d'aménagement

Appartenance à une chaîne (par rapport à un foyer individuel)

Ratio employés (équivalents temps plein) : lits

\section{Modèle 2 (prise en compte des caractéristiques de la circonscription sanitaire), RC ajusté (IC à $95 \%)$}

Modèle 3 (explicatif (prise en compte des caractéristiques du foyer), RC ajusté (IC à $95 \%)$

Remarque : COVID-19 = maladie à coronavirus 2019; IC = intervalle de confiance; RC ajusté = rapport de cotes ajusté pour tenir compte de toutes les autres covariables dans le modèle; Réf. = catégorie de référence; SLD = soins de longue durée.

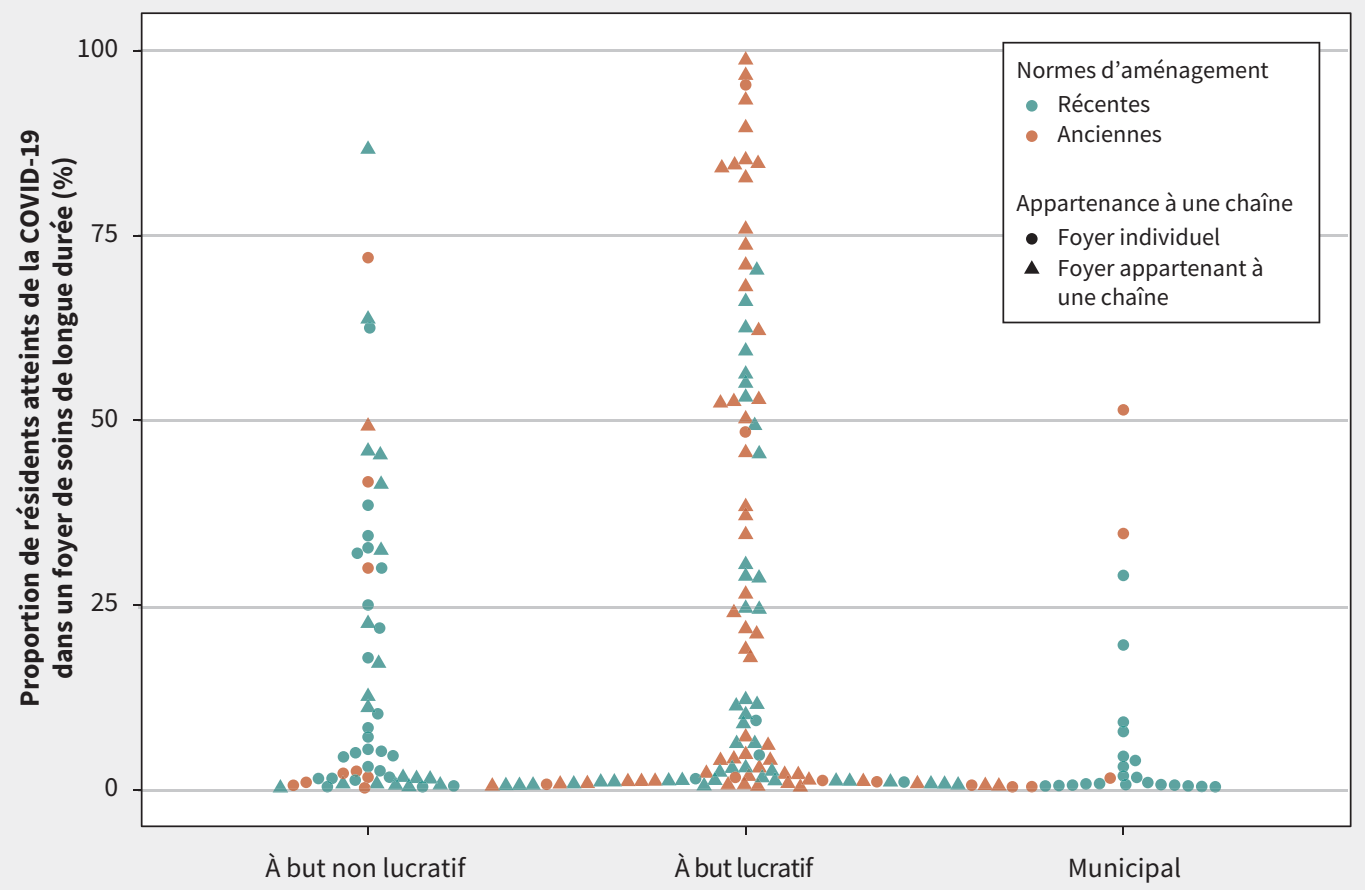

Statut financier

Figure 1 : Distribution de l'ampleur des éclosions de maladie à coronavirus 2019 (COVID-19) dans les foyers de soins de longue durée en fonction du statut financier. 
Tableau 4 : Ampleur* des éclosions de COVID-19 dans foyers de SLD en fonction du statut financier

Variable

Modèle 1

(statut financier

seulement),

RC ajusté (IC à $95 \%$ )
Modèle 2 (prise en compte des caractéristiques de la circonscription sanitaire), RC ajusté (IC à $95 \%$ )
Modèle 3 (explicatif) (prise en compte des caractéristiques du foyer), RC ajusté (IC à $95 \%$ )

\section{Statut financier}

À but non lucratif (Réf.)

À but lucratif

$$
\begin{aligned}
& 1,83(1,18-2,84) \\
& 0,60(0,28-1,30)
\end{aligned}
$$

$0,96(0,57-1,61)$

$0,85(0,40-1,82)$

\section{Caractéristiques de la circonscription sanitaire}

Incidence cumulative de la COVID-19 dans la circonscription sanitaire (1 cas par 1000 habitants)

Nombre d'habitants

$$
\begin{aligned}
& \geq 500000 \text { (Réf.) } \\
& 10000-499999 \\
& <10000 \text { (rural) }
\end{aligned}
$$

$0,65(0,33-1,24)$
$0,85(0,22-3,28)$
$0,55(0,30-0,99)$

$0,53(0,15-1,83)$

\section{Caractéristiques du foyer de SLD}

Nombre de résidents (par unité de 50)

Anciennes normes d'aménagement

Appartenance à une chaîne (par rapport à un foyer individuel)

Ratio employés (équivalents temps plein) : lits

Remarque : COVID-19 = maladie à coronavirus 2019; IC = intervalle de confiance; RC ajusté = rapport de cotes ajusté pour tenir compte de toutes les autres covariables dans le modèle; Réf. = catégorie de référence; $S L D=$ soins de longue durée.

${ }^{*}$ Nombre de résidents atteints de la COVID-19 dans les foyers de SLD en éclosion.

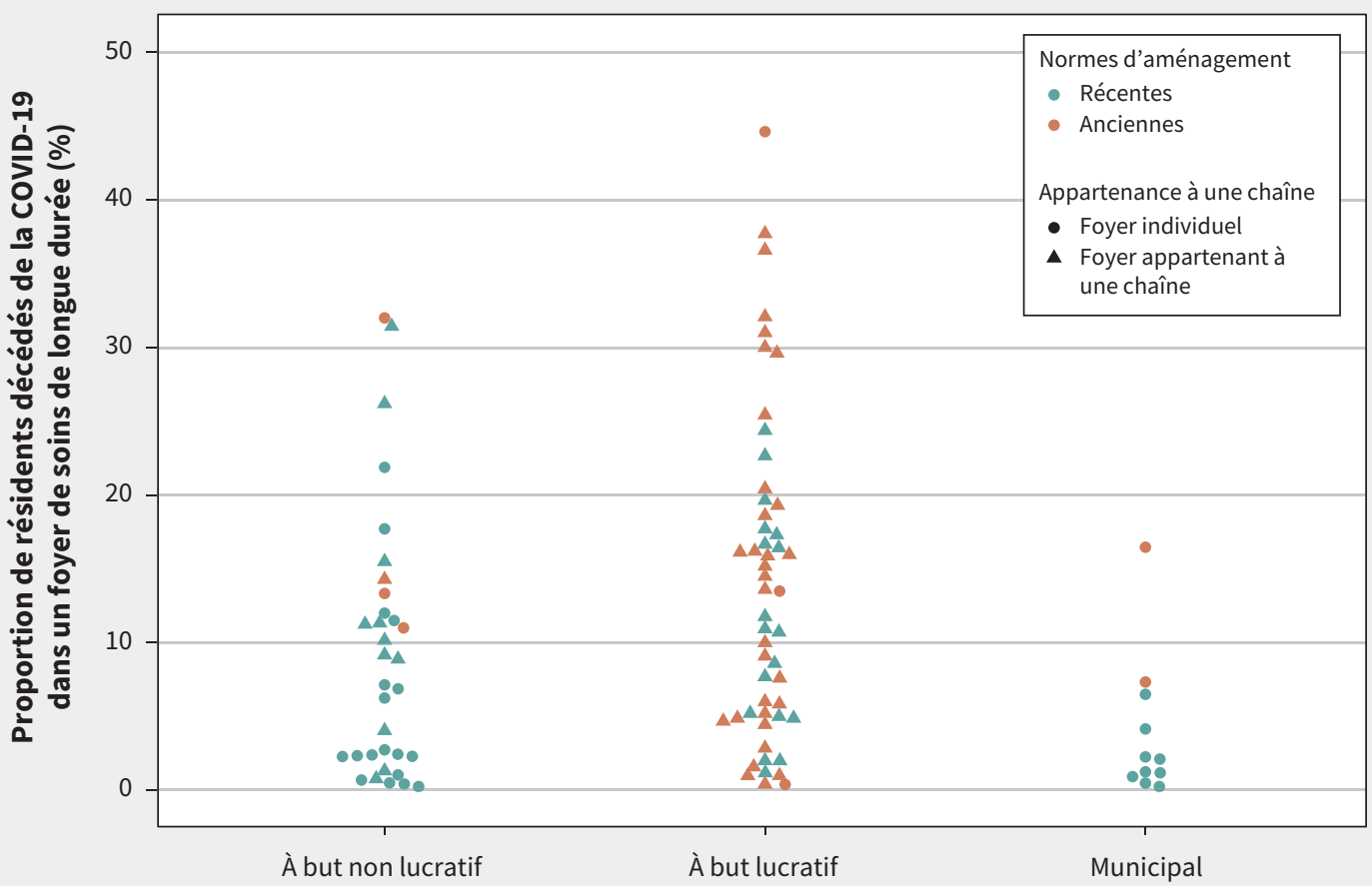

Statut financier

Figure 2 : Distribution du nombre de décès de résidents liés à la maladie à coronavirus 2019 (COVID-19) dans les foyers de soins de longue durée en fonction du statut financier. 


\begin{tabular}{|c|c|c|c|}
\hline Variable & $\begin{array}{c}\text { Modèle } 1 \\
\text { (statut financier } \\
\text { seulement), } \\
\text { RC ajusté } \\
\text { (IC à } 95 \% \text { ) }\end{array}$ & $\begin{array}{c}\text { Modèle } 2 \\
\text { (prise en compte des } \\
\text { caractéristiques de la } \\
\text { circonscription } \\
\text { sanitaire), } \\
\text { RC ajusté (IC à } 95 \% \text { ) }\end{array}$ & $\begin{array}{c}\text { Modèle } 3 \text { (explicatif } \\
\text { (prise en compte des } \\
\text { caractéristiques du } \\
\text { foyer), } \\
\text { RC ajusté (IC à } 95 \% \text { ) }\end{array}$ \\
\hline \multicolumn{4}{|l|}{ Statut financier } \\
\hline À but non lucratif (Réf.) & - & - & - \\
\hline À but lucratif & $1,67(0,99-2,79)$ & $1,78(1,03-3,07)$ & $0,82(0,44-1,54)$ \\
\hline Municipal & $0,50(0,19-1,29)$ & $0,54(0,20-1,49)$ & $0,73(0,28-1,88)$ \\
\hline \multicolumn{4}{|l|}{ Caractéristiques de la circonscription sanitaire } \\
\hline $\begin{array}{l}\text { Incidence cumulative de la COVID-19 dans la circonscription } \\
\text { sanitaire ( } 1 \text { cas par } 1000 \text { habitants) }\end{array}$ & - & $1,77(0,47-6,60)$ & $1,44(0,81-2,55)$ \\
\hline \multicolumn{4}{|l|}{ Nombre d'habitants } \\
\hline$\geq 500000$ (Réf.) & - & - & - \\
\hline $10000-499999$ & - & $0,62(0,26-1,47)$ & $0,51(0,25-1,05)$ \\
\hline$<10000$ (rural) & - & $0,72(0,12-4,25)$ & $0,40(0,08-1,89)$ \\
\hline \multicolumn{4}{|l|}{ Caractéristiques du foyer de SLD } \\
\hline Nombre de résidents (par unité de 50) & - & - & $0,81(0,70-0,95)$ \\
\hline Anciennes normes d’aménagement & - & - & $2,08(1,28-3,36)$ \\
\hline Appartenance à une chaîne (par rapport à un foyer individuel) & - & - & $1,89(1,00-3,59)$ \\
\hline Ratio employés (équivalents temps plein) : lits & - & - & $0,84(0,09-8,75)$ \\
\hline
\end{tabular}

Dans le modèle explicatif entièrement corrigé, l'association entre le nombre de décès de résidents et le statut « à but lucratif » était moins forte (RR ajusté 0,82 ; IC à $95 \% 0,44-1,54$ ). Le risque lié au nombre total de décès de résidents a toutefois été associé de façon significative avec l'application des anciennes normes d'aménagement (RR ajusté 2,08; IC à $95 \% 1,28-3,36$ ) et l'appartenance à une chaîne (RR ajusté 1,89; IC à $95 \%$ 1,00-3,59). Le nombre total de résidents actifs constituait pour sa part un facteur de protection (RR ajusté 0,81; IC à $95 \% 0,70-0,95$; par 50 lits).

\section{Analyses de sensibilité}

Pour chacun des 3 paramètres, les 3 modèles ont été réanalysés de nouveau en remplaçant les normes d'aménagement appliquées par l'occupation moyenne des chambres. Les effets estimés étaient semblables en ce qui concerne les associations avec le statut financier (annexe 1, tableau S2).

\section{Interprétation}

La présente étude sur les 623 foyers de SLD en Ontario (Canada) a conclu que les probabilités d'une éclosion de COVID-19 dans un foyer sont associées à l'incidence de la COVID-19 dans la circonscription sanitaire entourant le foyer, au nombre total de résidents actifs dans l'établissement et à l'application des anciennes normes d'aménagement, mais pas au statut "à but lucratif ».
Parmi les foyers où une éclosion a été confirmée, le statut «à but lucratif » a été associé à une ampleur des éclosions 1,96 fois $(1,26-3,05)$ plus élevée et à un nombre de décès de résidents liés à la COVID-19 1,78 fois (1,03-3,07) plus élevé que le statut «à but non lucratif » en tenant compte des caractéristiques des circonscriptions sanitaires. Les foyers municipaux ont obtenu les meilleurs résultats dans toutes les comparaisons. En général, les contributions des municipalités à ces foyers avantagent ces derniers sur le plan de la dotation en personnel et des dépenses en immobilisations (annexe 1, tableau S3) ${ }^{13,30}$.

L'association significative entre le risque d'éclosion dans un foyer de SLD et l'incidence de la COVID-19 dans la circonscription sanitaire entourant celui-ci est cohérente avec de nouvelles études montrant que le personnel de ces établissements constitue un important vecteur de transmission du SRAS-CoV- $2^{5,34}$. Durant la pandémie de COVID-19, la plupart des foyers de SLD sont devenus relativement clos en raison des mesures de restriction appliquées aux visites et aux transferts de résidents : des travailleurs infectés ont donc probablement été à l'origine de nombreuses éclosions, bien que la responsabilité ne leur incombe pas $^{35}$. Selon une étude antérieure sur les éclosions de COVID-19 dans les foyers de SLD en Ontario, un retard dans le dépistage des infections chez le personnel est un prédicteur significatif de futurs décès de résidents ${ }^{34}$. Il est possible que les protocoles de dépistage en place ne permettent pas de cibler les employés infectés n'ayant que peu ou pas de symptômes, et que d'autres 
continuent de travailler même s'ils sont malades parce qu'ils gagnent peu ou n'ont pas de congés de maladie ${ }^{6,8,36}$. Par ailleurs, bon nombre d'entre eux ont un emploi à temps partiel et travaillent dans plusieurs établissements de soins de santé, une situation qui a été associée à la transmission du SRAS-CoV-2 entre les foyers de $S L D^{5,37}$. L'association observée dans la présente étude entre le nombre de résidents actifs dans un foyer et le risque d'éclosion pourrait s'expliquer par le fait qu'un plus grand foyer nécessite plus de personnel, ce qui augmente le nombre de vecteurs potentiels d'infection ${ }^{38}$.

Dans la présente étude, l'association entre le statut « à but lucratif » et le nombre de cas de COVID-19 et de décès de résidents lors des éclosions semble largement attribuable à la prévalence élevée de normes d'aménagement désuètes dans ces foyers (ne dépassant pas les normes de 1972) ainsi qu'à leur appartenance à des chaînes : ces 2 facteurs menant à une plus grande transmission de la COVID-19. Ces résultats sont cohérents avec ceux d'études antérieures ayant relevé des associations faibles et non uniformes entre le statut «à but lucratif » des foyers et certains résultats non favorables, dont un risque accru d'infection chez les résidents ${ }^{11,12,39-41}$.

L'utilisation d'un modèle hiérarchique a permis de constater que les associations favorisant les foyers municipaux et à but non lucratif étaient moins fortes lorsque les normes d'aménagement en vigueur dans les foyers étaient prises en considération. Les normes plus récentes imposent des chambres plus grandes et plus privées ainsi que des aires communes autonomes et moins fréquentées, tandis que les anciennes normes d'aménagement prévoyaient des chambres partagées et des aires communes centralisées, permettant des interactions entre tous les résidents (annexe 1, tableau S1). En plus d'améliorer la qualité de vie des résidents, les plus récentes normes favorisent la prévention et le contrôle des infections en limitant le risque d'infection dans les chambres des résidents et entre les différentes zones d'un établissement ${ }^{31}$. De plus, les grands foyers avec davantage de résidents actifs semblent être mieux protégés en ce qui concerne l'ampleur des éclosions et le nombre de décès de résidents, ce qui pourrait s'expliquer par le fait que, conformément aux récentes normes, les aires d'habitation des résidents dans ces foyers sont autonomes et ne peuvent accueillir plus de 40 résidents.

La crise engendrée par la COVID-19 dans les foyers de SLD au Canada a mis en évidence des problèmes de longue date quant au financement, à l'exploitation et à la réglementation de ces établissement ${ }^{43}$. Des demandes pour une réforme des soins de longue durée se font déjà entendre, visant entre autres à éliminer les établissements privés à but lucratif de ce secteur ${ }^{8,44}$. Selon la présente étude, l'incidence de la COVID-19 dans la circonscription sanitaire entourant un foyer et la taille de celui-ci seraient d'importants facteurs de risque d'éclosion de la COVID-19, mais pas le statut "à but lucratif ", qui serait plutôt un important facteur de risque de transmission de la maladie après une éclosion puisqu'en général, ces foyers appliquent des normes d'aménagement désuètes et appartiennent à une chaîne. Or, force est de constater que ce ne sont pas tous les foyers à but lucratif qui affichent les pires résultats, mais bien ceux qui appliquent les anciennes normes d'aménagement, ce qui porte à croire que ces établissements n'ont pas été modernisés ou rénovés. Maintenant que différents gouvernements - dont celui de l'Ontario - se sont engagés à réclamer des commissions et des enquêtes indépendantes sur leurs systèmes de SLD, il est essentiel que les recommandations et les modifications tiennent compte de toutes les causes à l'origine de la crise actuelle, dont la nécessité de financer des projets d'immobilisations pour moderniser ou reconstruire les foyers plus anciens ${ }^{45}$.

\section{Limites de l'étude}

Une limite de la présente étude est le manque de données à l'échelle des résidents des foyers de SLD telles que leurs caractéristiques sociodémographiques et cliniques. Toutefois, puisque le processus d'admission dans les établissements ontariens est centralisé, les différences entre les effectifs des foyers à but lucratif, à but non lucratif et municipaux ne devraient pas être trop grandes. Puisque l'étude portait sur l'ensemble des foyers de SLD en Ontario, la taille de l'échantillon était maximale : aucun calcul de la taille de l'échantillon nécessaire n'a été effectué en amont. Des simulations post-hoc des éclosions fondées sur la distribution des cas observés dans les foyers ont toutefois confirmé que les analyses avaient un pouvoir de détection suffisamment grand pour détecter des différences entre l'ampleur des éclosions de COVID-19 dans les 3 types de foyers. Les données concernant la dotation en personnel étaient limitées au nombre d'équivalents temps plein assigné à chaque foyer : elles ne sont donc pas nécessairement représentatives du nombre d'employés travaillant dans un foyer à un moment précis. Il a également été impossible de déterminer si la prestation de soins dans les foyers à but non lucratif était sous-traitée à des organismes à but lucratif. De plus, les valeurs d'incidence de la COVID19 ont pu être imprécises dans le cas des foyers à la frontière de plusieurs circonscriptions sanitaires aux incidences différentes.

À l'instar d'autres sources de données ayant été recueillies rapidement durant la pandémie de COVID-19, les données fournies par la Direction de l'inspection des foyers de soins de longue durée n'ont pas fait l'objet d'une révision indépendante et auraient donc pu être incomplètes. Étant donné que plusieurs éclosions étaient toujours en cours à la fin de l'étude, il y a pu y avoir une censuration en aval des données, c'est-à-dire que des résidents infectés toujours vivants à ce moment sont peut-être décédés plus tard. C'est également pourquoi il a été difficile d'étudier les éclosions ne touchant que les employés qui auraient pu finir par se transmettre parmi les résidents. Il n'a pas été possible de tenir compte des changements observés au fil du temps en ce qui concerne l'accès à de l'équipement de protection individuelle dans les foyers de même que les changements dans les politiques provinciales sur les mesures de prévention et de contrôle des infections, le dépistage du SRAS-CoV-2 et les déplacements des employés entre les foyers. Ces facteurs seraient toutefois venus confirmer les résultats observés. Finalement, les taux de dépistage du SRAS-CoV-2 n'ont pas été pris en considération, mais puisque les taux de létalité associés à celuici et à la COVID-19 étaient similaires dans les 3 types de foyers de SLD, on peut supposer que les taux de dépistage de cas étaient également semblables. 


\section{Conclusion}

La présente étude a montré que le risque d'éclosion de COVID-19 dans un foyer de SLD était lié au taux d'incidence de la maladie dans la circonscription sanitaire entourant le foyer, au nombre total de lits dans l'établissement et à l'application des anciennes normes d'aménagement plutôt qu'au statut "à but lucratif ». L'étude a toutefois associé les foyers à but lucratif à des éclosions de COVID-19 de plus grande ampleur et à un nombre plus élevé de décès de résidents comparativement aux foyers municipaux et à but non lucratif. Ces conclusions s'expliquent par le nombre élevé de foyers à but lucratif appliquant les anciennes normes d'aménagement et appartenant à une chaîne de foyers. La pandémie de COVID-19 a mis en évidence des problèmes de longue date par rapport au financement, à l'exploitation et à la réglementation des foyers de SLD ${ }^{45}$. Pendant que les systèmes de santé tentent de préparer les foyers en vue des prochaines vagues de la pandémie de COVID-19 et que d'autres veillent à la reddition de comptes et cherchent des solutions pour remédier à la crise dans le secteur des SLD, il est impératif d'étudier toutes les explications possibles aux différents résultats de la COVID-19 observés dans les foyers de SLD.

\section{Références}

1. Barnett ML, Grabowski DC. Nursing homes are ground zero for COVID-19 pandemic. JAMA Health Forum 24 mars 2020. Accessible ici : https://jamanetwork. com/channels/health-forum/fullarticle/2763666 (consulté le $1^{\text {er }}$ mai 2020).

2. Hsu AT, Lane N, Sinha SK, et al. Understanding the impact of COVID-19 on residents of Canada's long-term care homes - ongoing challenges and policy responses. Londres (Royaume-Uni) : International Long-Term Care Policy Network; 24 mars 2020, modifié le 4 juin 2020. Accessible ici : https://tccovid.org/ wp-content/uploads/2020/06/LTCcovid-country-reports_Canada_June-4-2020

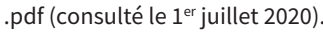

3. Pandemic experience in the long-term care sector: How does Canada compare with other countries? Ottawa: Canadian Institute for Health Information; 2020. Accessible ici : www.cihi.ca/sites/default/files/document/covid-19-rapid

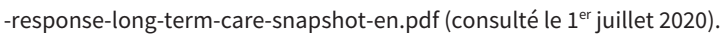

4. D’Adamo H, Yoshikawa T, Ouslander JG. Coronavirus disease 2019 in geriatrics and long-term care: the ABCDs of COVID-19. J Am Geriatr Soc 2020;68:912-7.

5. McMichael TM, Currie DW, Clark S, et al. Epidemiology of COVID-19 in a long-term care facility in King County, Washington. N Engl J Med 2005;382:2005-11.

6. Hoxha A, Wyndham-Thomas C, Klamer S, et al. Asymptomatic SARS-CoV-2 infection in Belgian long-term care facilities. Lancet Infect Dis 3 juillet 2020; S1473-3099(20)30560-0.

7. Ouslander JG. Coronavirus disease 19 in geriatrics and long-term care: an update. J Am Geriatr Soc 2020;68:918-21.

8. Holroyd-Leduc JM, Laupacis A. Continuing care and COVID-19: a Canadian tragedy that must not be allowed to happen again. CMAJ 2020;192:E632-3.

9. Jabbar A, Raza D. Let's keep profit out of long-term care. The Toronto Star 6 mai 2020. Accessible ici : http://www.thestar.com/opinion/contributors /2020/05/06/lets-keep-profit-out-of-long-term-care.html (consulté le 17 mai 2020).

10. Ronald LA, McGregor MJ, Harrington C, et al. Observational evidence of forprofit delivery and inferior nursing home care: When is there enough evidence for policy change? PLoS Med 2016;13:e1001995.

11. Comondore VR, Devereaux PJ, Zhou Q, et al. Quality of care in for-profit and not-for-profit nursing homes: systematic review and meta-analysis. BMJ 2009;339:b2732.

12. Hillmer MP, Wodchis WP, Gill SS, et al. Nursing home profit status and quality of care: is there any evidence of an association? Med Care Res Rev 2005;62:139-66.

13. McGregor MJ, Cohen M, McGrail, K et al. Staffing levels in not-for-profit and forprofit long-term care facilities: Does type of ownership matter? CMAJ 2005;172:645-9.
14. Harrington $\mathrm{C}$, Olney $\mathrm{B}$, Carrillo $\mathrm{H}$, et al. Nurse staffing and deficiencies in the largest for-profit nursing home chains and chains owned by private equity companies. Health Serv Res 2012;47:106-28.

15. Hsu AT, Berta W, Coyte PC, et al. Staffing in Ontario's long-term care homes: differences by profit status and chain ownership. Can J Aging 2016;35:175-89.

16. McGregor MJ, Cohen M, Stocks-Rankin CR, et al. Complaints in for-profit, nonprofit and public nursing homes in two Canadian provinces. Open Med 2011;5: e183-92.

17. Tanuseputro $P$, Chalifoux $M$, Bennett, $C$ et al. Hospitalization and mortality rates in long-term care facilities: Does for-profit status matter? J Am Med Dir Assoc 2015;16:874-83.

18. Hirth RA, Grabowski DC, Feng Z, et al. Effect of nursing home ownership on hospitalization of long-stay residents: an instrumental variables approach. Int $J$ Health Care Finance Econ 2014;14:1-18.

19. McGregor MJ, Abu-Laban RB, Ronald LA, et al. Nursing home characteristics associated with resident transfers to emergency departments. Can J Aging 2014;33:38-48.

20. McGregor MJ, Tate RB, McGrail KM, et al. Care outcomes in long-term care facilities in British Columbia, Canada. Does ownership matter? Med Care 2006;44:929-35.

21. Grabowski DC, Feng Z, Hirth R, et al. Effect of nursing home ownership on the quality of post-acute care: an instrumental variables approach. $J$ Health Econ 2013;32:12-21.

22. Damián J, Pastor-Barriuso R, García-López FJ, et al. Facility ownership and mortality among older adults residing in care homes. PLOS One 2019;14: e0197789.

23. Castle NG, Wagner LM, Ferguson-Rome JC, et al. Nursing home deficiency citations for infection control. Am J Infect Control 2011;39:263-9.

24. Castle N, Wagner L, Ferguson J, et al. Hand hygiene deficiency citations in nursing homes. J Appl Gerontol 2014;33:24-50.

25. COVID-19 outbreak guidance for long-term care homes (LTCH). Toronto: Ontario Ministry of Health; 15 avril 2020. Accessible ici : www.health.gov.on.ca/ en/pro/programs/publichealth/coronavirus/docs/LTCH_outbreak_guidance.pdf (consulté le 5 mai 2020).

26. Benchimol El, Smeeth L, Guttmann A, et al. The Reporting of studies Conducted using Observational Routinely-collected health Data (RECORD) statement. PLoS Med 2015;12:e1001885.

27. von Elm E, Altman DG, Egger M, et al. Strengthening the Reporting of Observational Studies in Epidemiology (STROBE) statement: guidelines for reporting observational studies. BMJ 2007;335:806-8.

28. iPHIS resources. Toronto: Public Health Ontario; 2020. Accessible ici : www. publichealthontario.ca/en/diseases-and-conditions/infectious-diseases/ccm/ iphis (consulté le 17 mai 2020).

29. eHealth Ontario. Ontario Laboratories Information System (OLIS). Toronto: Ontario Health; 2020. Accessible ici : www.ehealthontario.on.ca/en/for-healthcare -professionals/ontario-laboratories-information-system-olis (consulté le 13 juillet 2020)

30. Daly T. Dancing the two-step in Ontario's long-term care sector: more deterrence-oriented regulation = ownership and management consolidation . Stud Polit Econ 2015;95:29-58.

31. Long-term care home design manual 2015. Toronto: Ontario Ministry of Health and Long-Term Care; 2015. Accessible ici : http://health.gov.on.ca/en/public/ programs/ltc/docs/home_design_manual.pdf (consulté le 22 mai 2020).

32. Postal code OM conversion file plus (PCCF+). Ottawa: Statistics Canada; 2020. Accessible ici : https://www150.statcan.gc.ca/n1/en/catalogue/82F0086X (consulté le 28 juin 2020).

33. Harrison XA. Using observation-level random effects to model overdispersion in count data in ecology and evolution. PeerJ 2014;2:e616.

34. Fisman D, Bogoch I, Lapointe-Shaw L, et al. Risk factors associated with mortality among residents with COVID-19 in long-term care facilities in Canada. JAMA Network Open 22 juillet 2020. doi: 10.1101/jamanetworkopen.2020 .15957 .

35. Chow EJ, Schwartz NG, Tobolowsky FA, et al. Symptom screening at illness onset of health care personnel with SARS-CoV-2 infection in King County, Washington. JAMA 2020;323:2087-89. 
36. Pollock AM, Clements L, Harding-Edgar L. Covid-19: why we need a national health and social care service. BMJ 2020;369:1465.

37. Van Houtven CH, DePasquale N, Coe NB. Essential Long-Term Care Workers Commonly Hold Second Jobs and Double- or Triple-Duty Caregiving Roles. J Am Geriatr Soc 27 avril 2020 [Cyberpublication avant impression]. doi: 10.1111/ jgs.16509.

38. Baldwin R, Chenoweth L, Dela Rama M, et al. Does size matter in aged care facilities? A literature review of the relationship between the number of facility beds and quality. Health Care Manage Rev 2017;42:315-27.

39. Konetzka RT, Spector W, Shaffer T. Effects of nursing home ownership type and resident payer source on hospitalization for suspected pneumonia. Med Care 2004;42:1001-8.

40. Chou SY. Asymmetric information, ownership and quality of care: an empirical analysis of nursing homes. J Health Econ 2002;21:293-311.
41. Spector WD, Selden TM, Cohen JW. The impact of ownership type on nursing home outcomes. Health Econ 1998;7:639-53.

42. Long-term care facility design manual. Toronto: Ontario Ministry of Health and Long-Term Care; 1999. Accessible ici : https://collections.ola.org/mon/ont/H/1999/ LTCdesign_manual.pdf

43. Grabowski DC, Mor V. Nursing home care in crisis in the wake of COVID-19. JAMA 22 mai 2020 [Cyberpublication avant impression]. doi: 10.1001/jama.2020.8524.

44. Lessons from a pandemic: union recommendations for transforming long-term care in Canada. North York (ON): Canadian Labour Congress; 2020. Accessible ici : http:// documents.clcctc.ca/sep/LongTermCare-Report-EN.pdf (consulté le 19 mai 2020).

45. Ontario announces independent commission into long-term care [media release]. Toronto: Ontario Ministry of Long-Term Care; 19 mai 2020. Accessible ici : https://news.ontario.ca/mltc/en/2020/05/ontario-announces-independent -commission-into-long-term-care.html (consulté le 20 mai 2020).
Intérêts concurrents : Andrew Costa indique qu'il est titulaire d'une chaire de recherche de l'Institut Schlegel de l'Université McMaster. Cette chaire est financée par la famille Schlegel (qui est propriétaire de Schlegel Villages [une chaîne de foyers de retraite et de soins de longue durée à but lucratif] ainsi que d'autres entreprises non apparentées). Aucun autre intérêt concurrent déclaré.

Cet article a été révisé par des pairs.

Affiliations : Division de médecine interne générale et de gériatrie (Stall), Système de santé Sinaï et Réseau universitaire de santé; Institut de recherche du Women's College (Stall, Rochon), Hôpital Women's College; Département de médecine (Stall, Rochon) et Institut des politiques, de la gestion et de l'évaluation de la santé (Stall, Rochon), Université de Toronto, Toronto (Ontario); Département des méthodes, des données et de l'incidence de la recherche en santé (Jones, Costa), Université McMaster, Hamilton (Ontario); Prévention et contrôle des infections (Brown), Santé publique Ontario; École de santé publique Dalla Lana (Brown), Université de Toronto, Toronto (Ontario); Chaire de l'Institut Schlegel en épidémiologie clinique et en vieillissement (Costa), Université McMaster; Centre de soins intégrés (Costa), Système de soins de santé St-Joseph, Hamilton (Ontario).

Collaborateurs : Nathan Stall, Aaron Jones, Kevin Brown et Andrew Costa ont contribué à l'élaboration et à la conception de l'étude. Tous les auteurs ont recueilli, analysé ou interprété les données. Aaron Jones a procédé aux analyses statistiques. Nathan Stall a rédigé la première version du manuscrit. Tous les auteurs ont révisé de façon critique le contenu intellectuel important du manuscrit et assument l'entière responsabilité de tous les aspects du travail.

\section{Financement : Cette étude n'a pas été financée.}

Partage des données : Le protocole de l'étude et les codes statistiques seront fournis sur demande par les auteurs (courrier électronique nathan.stall@sinaihealth.ca). À noter que certains programmes informatiques pourraient reposer sur des modèles de codage ou des macros inaccessibles ou nécessitant des modifications. L'ensemble de données est hébergé de façon sécuritaire par la Division de la planification de la capacité et de l'analytique du ministère de la Santé de l'Ontario. Des ententes sur le partage des données en empêchent la publication.

Remerciements : Les auteurs remercient chaleureusement Michael Hillmer, Kamil Malikov et Sping Wang de la Division de la planification de la capacité et de l'analytique du ministère de la Santé de l'Ontario pour leur aide avec l'acquisition, l'interprétation et l'analyse des données. Nathan M. Stall reçoit un soutien financier du Programme de formation des cliniciens-chercheurs Eliot Phillipson du Département de médecine et du Programme de cliniciens-chercheurs de l'Université de Toronto, et est titulaire d'une bourse d'études supérieures du Canada Vanier. Paula A. Rochon est titulaire de la chaire de RTOERO en médecine gériatrique de l'Université de Toronto. Andrew P. Costa est titulaire de la chaire de recherche de l'Institut Schlegel en épidémiologie clinique et en vieillissement de l'Université McMaster.

Déclaration d'intérêts : Nathan Stall est rédacteur associé pour le CMAJ, mais n'a pas participé au processus ayant mené au choix de cet article.

Accepté : 14 juillet 2020

Correspondance : Nathan Stall, nathan.stall@sinaihealth.ca 